\title{
CALIBRATION OF A MEASURING DEVICE TO DETERMINE SPECIFIC SATURATION MAGNETIZATION BY A SPECIAL COIL
}

\begin{abstract}
Michal Ulvr *
Specific saturation magnetization $\sigma_{S}$ is an important parameter of ferromagnetic materials. It is in principle independent of the structure and shape of the sample. There are two ways to calibrate devices for determining $\sigma_{S}$ : the standard method, using a calibrated magnetic moment standard, or an alternative method, using a special moment coil. This paper presents a calibration method used at Czech Metrology Institute for calibration of Koerzimat 1.096 using a special moment coil. Special attention is given to a description of the special moment coils used for Koerzimat 1.096 calibration, and to analysis of sources of uncertainty. Calibration expanded uncertainty of 0.4 up to $0.6 \%$ can be achieved using this method.
\end{abstract}

K e y w ords: calibration, magnetic moment, magnetic moment standard, moment coil, saturation magnetization

\section{INTRODUCTION}

In many fields of metal processing (eg metallurgy, magnet and cemented carbide manufacturing, and magnetic material control), the saturation magnetization provides information on the properties and the composition of the material, and also on the quality of the material. Measuring devices for determining specific saturation magnetization $\sigma_{S}$, such as Koerzimat 1.096 and Sigmameter, work on the principle of magnetic moment measurement, according to [1]. The weight of the sample is first determined with precision scales. The sample is then positioned in the air gap of a saturation magnet (a shielded permanent magnet with a large air gap) and is pulled out. The magnetic moment is measured by means of a Helmholtz measuring coil, arranged in the air gap of the saturation magnet, and a fluxmeter. The weight-specific saturation magnetization $\sigma_{S}$ is then calculated from the magnetic moment ratio to weight. According to [1], devices of this kind should be calibrated by a calibrated magnetic moment standard of suitable dimensions made of $99.99 \%$ pure nickel. We present an alternative method for Koerzimat 1.096 calibration using a special moment coil.

\section{THEORY}

In calibration method using a magnetic moment standard the test systems can be calibrated to higher resolution without loss of accuracy by using nickel rather than iron. Since nickel is chemically stable, it will not rust or corrode, thus avoiding attendant changes in magnetic characteristics. High-purity nickel is well-suited for use as a magnetic reference material. A disadvantage of nickel, however, is that it is a very soft metal and thus requires reasonably careful handling. As long as it is not physically damaged, however, its properties do not change. If it is crushed or otherwise damaged, it can be ground or machined to a new shape and, if properly annealed for stress-relief, can be certified again to its original magnetic specifications.

The principle of the calibration method for Koerzimat 1.096 using a magnetic moment standard made from nickel consists in comparing the calibrated value of the magnetic moment standard with the value measured according to [1]. The magnetic moment standard must have proper dimensions (it usually has the shape of a disc). The magnetic moment of the nickel standard can be determined $e g$ by a sampling technique using an absolute magnetometer based on the Faraday method (developed at NIST). The purity of the nickel at a level of least $99.99 \%$ must be certified eg by a chemical laboratory. The advantage of this method is its simplicity. The disadvantage is that the magnetic moment standard has only one nominal value, so if you want to calibrate the device in more than in one measuring point, for example in five measuring points, you need to have five magnetic moment standards with different nominal values.

Calibration method, which has been used successfully at the Czech Metrology Institute is based on a special moment coil. Figure 1 presents a schematic diagram of the calibration aparatus. The moment coil, powered by current $I$ and inserted into the measuring Helmholtz coils (inside the saturation magnet air gap) is to simulate a sample of ferromagnetic material. The magnetic moment of this coil is determined by its current $I$ with $K_{S}$ being the proportionality constant. Using this coil, the set saturation magnetization value can be found as

$$
\sigma_{S c}=\mu_{0} \frac{K_{S} I}{m}=\mu_{0} \frac{K_{S} U_{N}}{m R_{n}},
$$

where $\mu_{0}$ is the magnetic constant $\left(4 \pi \times 10^{-7} \mathrm{H} / \mathrm{m}\right), m$ is the specimen weight, and $U_{N}$ is the voltage drop on the standard resistor $R_{n}$ measured by a digital multimeter (DMM).

* Department of Electromagnetic Quantities, Czech Metrology Institute, V Botanice 4, 15072 Prague 5, Czech Republic, mulvr@cmi.cz 


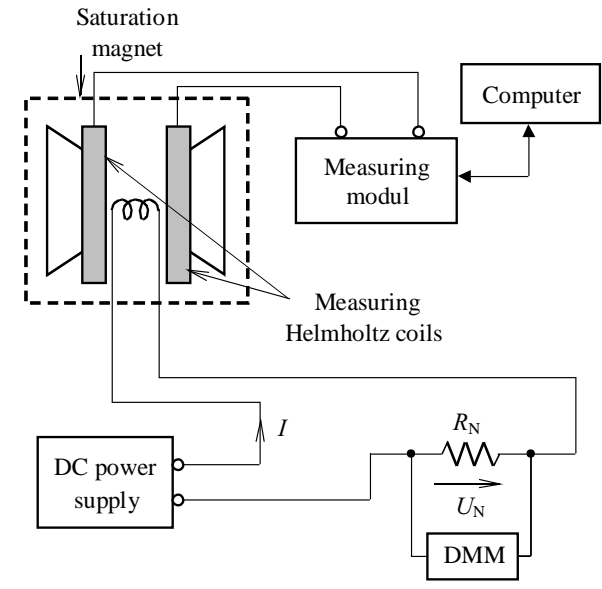

Fig. 1. Schematic diagram of Koerzimat 1.096 calibration using a special moment coil
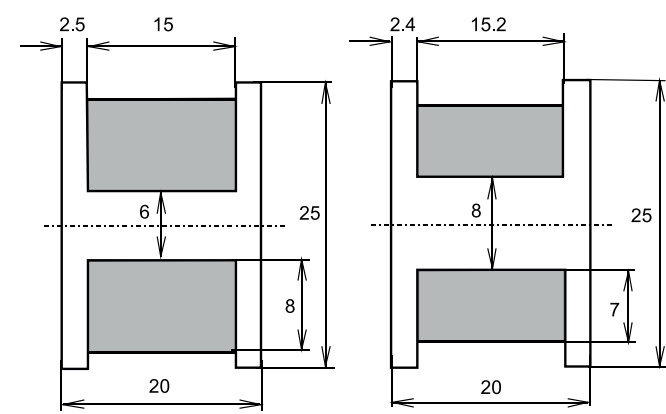

Fig. 2. Cross-section of the frame of the special coils EP 10/97 and EP 9/97 for calibration of Koerzimat 1.096

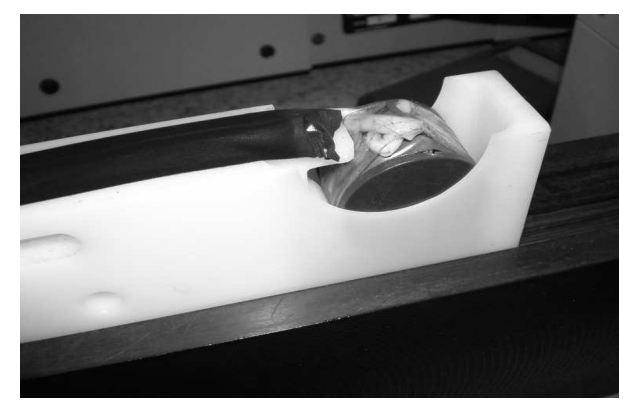

Fig. 3. Detail of moment coil EP 10/97 inserted into the test piece slide

\section{SPECIAL MOMENT COILS}

The system of axially symmetric conductive loops with area turns $N A$ passed by current $I$ can be described by the magnetic moment $m=I N A$, which is a vector quantity describing the magnetic dipole of the moment coil. In the real case of a system of loops, an accurate description is a series of multipoles with multipole constants $p_{0}$, $p_{1}, p_{2}, \ldots, p_{n}$. A multipole with constant $p_{0}$ equals zero because of the absence of a magnetic monopole. A multipole with constant $p_{1}$ is a magnetic dipole; $p_{1}$ is the area turns for a coil. A multipole with constant $p_{2}$ is a quadrupole that equals zero as do all even multipole constants due to the symmetry of the system. The third multipole, with a constant of $p_{3}$, is an octupole, etc. The influence of the higher multipoles declines with distance.
The influence can be significant in distances comparable with $5-10$ times the dimensions of the coil. To suppress higher multipoles, especially $p_{3}$ of symmetrical cylindrical windins we choose the ratio of the coil's length to its diameter $\sqrt{3} / 2$, [2]. This ratio can also be used for the multi-layer search coil.

With a coil having non compensated octupole constant - for example moment coil with small ratio of the coils length to diameter - the measurement error caused by an octupole placed in a short solenoid (not enough homogeneous external field) can be estimated to a few percent. If a compensated moment coil is placed into the magnetic field with a better homogeneity ( $e g$ Helmholtz coils) then the measurement error due to the octupole is reduced to a few tenths of a percent or less. Moreover, the moment coil with compensated octupole constant when placed into a homogeneous external magnetic field is also less sensitive to fabrication inaccuracies of a real coil.

Because the test piece slide for the saturation magnet has a limited space, the dimensions (and thus the area turns) of the moment coil are also limited. Two special moment coils EP 9/97 and EP 10/97 were designed with a cylindrical frame and with a suppressed octupole. The frame was made from textit, and is $25 \mathrm{~mm}$ in length and about $20 \mathrm{~mm}$ in diameter, as shown in Fig. 2. The idea was raised of using silver wire for the coil winding. Silver has a smaller resistivity value than copper, which means that when there are the same number of turns, the value of coil's power should be smaller than when using copper wire. Finally, after making calculations, we decided to use copper wire, because the power value would be only a few percent smaller, and would not justify the higher cost of silver. The winding of both coils is wound with enameled copper wire $0.5 \mathrm{~mm}$ in diameter. The moment coil constant value $(0.092682 \pm 0.000074) \mathrm{m}^{2}$ for EP $10 / 97$ and $(0.066378 \pm 0.000053) \mathrm{m}^{2}$ for EP $9 / 97$ was determined by a method with variable mutual inductance [3]. The maximum current value through the coils can be $1 \mathrm{~A}$ for a short time (eg few minutes).

\section{UNCERTAINTY ANALYSIS}

The A-type of uncertainty of the measurement is calculated from

$$
U_{s A}=\sqrt{\frac{\sum_{i=1}^{n}\left(x_{i}-\bar{x}\right)^{2}}{n(n-1)}}, \quad \bar{x}=\frac{1}{n} \sum_{i=1}^{n} x_{i} .
$$

where $n$ is the total number of measurements and $\bar{x}$ is the arithmetic mean of the individual measured values $x_{i}$

The B-type uncertainty of the calibration method has several components, as follows

$$
U_{s B}=\sqrt{u_{S C}^{2}+u_{V}^{2}+u_{R}^{2}+u_{\varphi}^{2}+u_{T}^{2}+u_{H}^{2}},
$$

where $u_{S C}$ is the standard uncertainty of the moment coil constant, $u_{V}$ is the uncertainty of the voltage value measured on the standard resistor $R_{N}, u_{R}$ is the uncertainty 
of the standard resistor, $u_{\varphi}$ is the uncertainty of the directional dependence measurement of the moment coil, $u_{T}$ is the uncertainty of the influence of the moment coil winding temperature, and $u_{H}$ is the uncertainty of the influence of homogeneity inside the measuring Helmholtz coils in the saturation magnet air gap.

The uncertainty of the search coil constant determined by calibration with variable mutual inductance is $0.04 \%$. The uncertainty of the measured voltage on the standard resistor is dependent on the specification of the digital multimeter that is used. The value of $u_{V}$ can be of order of thousandths of one percent. The maximum uncertainty value of the standard resistor usually varies in units to tens of ppm, so it can be neglected, because the other uncertainties are essentially higher. The value of the output voltage impulse from the measuring Helmholtz coils depends on the direction in which the moment coil is inserted into the measuring Helmholtz coils inside the saturation magnet air gap. In principle, the value depends on $\cos \varphi$, where $\varphi$ is the angle between the axis of the moment coil and the axis of the measuring Helmholtz coils. The search coil must be set to the position where $\cos \varphi=1$, which is the maximum value from the measuring Helmholtz coils. For example, there is a difference of about $0.02 \%$ from the true value of the measured voltage impulse for $\varphi=1^{\circ}$, there is a difference of about $0.06 \%$ for $\varphi=2^{\circ}$, and so on. This means that the value of $u_{\varphi}$ can lie in the order of hundredths to tenths of one percent. The current through the moment coil causes the winding to heat up, and thus changes the area turns value. This means that the influence of the moment coil temperature winding must also be taken into account. The value of $u_{T}$ can lie in the order of hundredths to tenths of one percent. The influence of homogeneity inside the measuring Helmholtz coils is small, due to the suppressed octupole of the moment coil that is used, but its value is nonnegligible. The value of $u_{H}$ was determined as $0.08 \%$.

The total (combined) uncertainty of the calibration method is then calculated as $U_{s}=\sqrt{u_{s A}^{2}+u_{s B}^{2}}$.

\section{MEASUREMENT RESULTS}

The method described above was tested using Koerzimat 1.096 in the laboratory of Global Tungsten \& Powders, Czech Republic. The calibration was performed for the sets weight value of $2.5 \mathrm{~g}, 5 \mathrm{~g}$ and $10 \mathrm{~g}$. The moment coil EP 10/97 was used for calibration, and the results are presented in Tab. 1. The nominal current value through the moment coil was $0.5 \mathrm{~A}$ and $1 \mathrm{~A}$. The real current was measured as a voltage drop by the HP 34401A-type multimeter on the Guildline 9221-type standard resistor with a calibrated value of $(2.000038 \pm 0.000011) \Omega$. Ten measurements were performed for each weight value. The values presented in Tab. 1 are the arithmetic mean of these measurements.
Table 1. Result of simulations

\begin{tabular}{cccccc}
\hline \multicolumn{2}{c}{$\begin{array}{c}\text { Measured } \\
\text { Measured } \\
\text { Weight current }\end{array}$} & $\begin{array}{c}\sigma_{S c} \text { by } \\
\text { Koerzimat } \\
\text { sets by } \\
\text { moment } \\
\text { coil }\end{array}$ & $\begin{array}{c}\text { Relative } \\
\text { error of } \\
\text { measured of calibration } \\
\text { value }\end{array}$ & $\begin{array}{c}\text { Expanded } \\
\text { uncertainty } \\
\text { for } \mathrm{k}=2 \\
(\%)\end{array}$ & $\begin{array}{c}1.096 \\
(\mathrm{mT})\end{array}$ \\
\hline 2.5 & 0.52669 & 24.53 & 24.54 & 0.04 & 0.5 \\
5 & 0.51238 & 11.91 & 11.94 & -0.25 & 0.5 \\
5 & 1.00317 & 23.36 & 23.37 & -0.04 & 0.4 \\
10 & 0.51615 & 6.007 & 6.011 & -0.07 & 0.5 \\
\hline
\end{tabular}

\section{CONCLUSIONS}

An alternate method for calibration of measuring device for determining specific saturation magnetization has been proposed. The construction and the parameters of the special moment coils have been described, and an uncertainty analysis is also presented. When a method with a special moment coil is used, the calibration uncertainty depends mainly on the uncertainty of the moment coil constant, on the uncertainty of the measured current, which is measured as the voltage drop on the standard resistor, and on the uncertainty of the placement of the moment coil in the measuring Helmholtz coils in the saturation magnet air gap. The influence of the homogeneity inside the measuring Helmholtz coils and the influence of the moment coil winding temperature must also be taken into account. The advantage of this calibration method is that we can make a calibration for more than one value of $\sigma_{S c}$ with a single moment coil and by changing the current and weight value. A calibration expanded uncertainty value of 0.4 up to $0.6 \%$ can be achieved using this method.

\section{REFERENCES}

[1] IEC 60404-14: Methods of Measurement of the Magnetic Dipole Moment of a Ferromagnetic Material Specimen by the Withdrawal or Rotation Method.

[2] AFANASJEV, J. V. et al : Means for Measuring of Magnetic Fields (Sredstva izmerenij parametrov magnitnogo polja), Energia, Leningrad, 1979. (in Russian)

[3] ULVR, M. : A System for Calibration of Search Coils with Area Turns up to $100 \mathrm{~m}^{2}$, Proc. of CPEM 2012, July 2012.

[4] Institut Dr. Foerster, Koerzimat CS 1.096 [online], http://www.foerstergroup.de/fileadmin/MEDIA/DM/ Brochures-data_sheets/en/1096_GB_EN_REV0907.pdf.

[5] SHULL, R. D.-McMICHAEL, R. D.-SWARTZENDRUBER, L. J.-LEIGH, S. D.: Absolute Magnetic Moment Measurements of Nickel Spheres, Journal of Applied Physic 87 No. 9 (May 2000).

Received 7 January 2014

Michal Ulvr was born in Jablonec nad Nisou, Czech Republic, in 1983. He was awarded his master's degree in measurement techniques from the Czech Technical University in Prague in 2008. He has been working at the Czech metrology institute since 2008, and is now a metrologist in the Department of Electromagnetic Quantities of the Laboratory of Fundamental Metrology in Prague. His research interest is in metrology in the area of magnetic quantities. 